\title{
Fuchs endothelial corneal dystrophy and small eyes
}

\author{
Nicole Shao-Yuen Lim, ${ }^{1}$ John Males ${ }^{2}$ \\ ${ }^{1}$ Department of Ophthalmology, Sydney Eye Hospital, Sydney, Australia; ${ }^{2}$ The \\ University of Sydney, Save Sight Institute, Sydney Eye Hospital, Sydney, Australia
}

\begin{abstract}
Aim: To determine whether there is an association between Fuchs endothelial corneal dystrophy (FECD) and shorter axial length $(A L)$, shallower anterior chamber depth $(A C D)$ and higher spherical equivalent (SE). In addition, to evaluate whether there is a correlation between AL and severity of corneal decompensation in FECD, using corneal thickness as a proxy.

Design: Retrospective cohort study.

Methods: This was a single-centre study conducted in a cornea clinic in Sydney, Australia. Detailed clinical measurements of 91 eyes of 50 FECD patients were compared with 110 eyes of 55 controls. Main outcome measures included AL, ACD and SE. Other outcome measures included central corneal thickness, visual acuity, intraocular pressure and keratometry.

Results: Mean AL of FECD patients was $23.6 \mathrm{~mm}$ (standard deviation [SD] $\pm 0.9 \mathrm{~mm}$ ), compared with $24.7 \mathrm{~mm}$ (SD $\pm 1.8 \mathrm{~mm}$ ) for controls $1.1 \mathrm{~mm}$ difference [95\% confidence interval [Cl] 0.5-1.6], $p<0.001$, independent sample t-test); corresponding means for ACD were 3.0 and $3.3 \mathrm{~mm}(0.32 \mathrm{~mm}$ difference [95\% Cl 0.2-0.5], $p<0.001$, independent t-test). Eleven out of the 22 FECD patients with available refraction data had hypermetropic refraction compared with 16 out of 36 controls ( $p=0.68$, chi-squared test). The mean SE of FECD patients $(+0.10 D)$ was higher than controls $(-1.33 D)$ (1.4D difference [0.1-2.8], $p=0.04$, independent $t$-test). No statistically significant correlation was found between $A L$ and corneal thickness ( $p=0.28$, linear regression).

Conclusion: In this retrospective cohort study, a strong association was established between FECD and small eyes, with shorter AL and shallower ACD, compared with controls. These results have important implications for surgical planning, as shorter $A L$ and ACD in FECD patients likely contribute to their high risk of corneal decompensation following cataract surgery.
\end{abstract}

Keywords: anterior chamber depth, axial length, Fuchs endothelial corneal dystrophy, hypermetropia, hyperopia

Correspondence: John Males, Save Sight Institute, Sydney Eye Hospital, Level 12 Park House, 187 Macquarie St, Sydney NSW 2000, Australia.

E-mail:john.males@sydney.edu.au 


\section{Introduction}

Fuchs endothelial corneal dystrophy (FECD) is the most common corneal dystrophy and one of the leading indications for corneal transplant. ${ }^{1,2}$ The condition is characterized by progressive loss of endothelial cells and increase in extracellular matrix deposition at the level of Descemet's membrane. ${ }^{3}$ Although the condition has been well defined clinically, refractive profiles and axial lengths (ALs) of patients with FECD have yet to be adequately described.

Only two small reports have attempted this. The first was a study of 24 patients by Pitts and Jay, ${ }^{4}$ which suggested a tendency of FECD patients to be hypermetropic with short ALs. This was supported by a non-comparative series of 23 patients by Lowenstein et al. ${ }^{5}$ Drawbacks of this research, published over two decades ago, include the small sample size, inclusion of measurements taken post-transplant and significant proportion of subjects excluded from analysis due to absent data. These studies also used applanation ultrasound biometry for AL and anterior chamber depth (ACD) measurements. Non-contact optical biometry has since surpassed ultrasonography as the gold standard technique for biometric parameters, facilitating more accurate and reproducible measurements. ${ }^{6,7}$ Specifically, optical biometry has been found to be superior in eyes with short ALs, where there is potential for corneal compression with applanation ultrasound. ${ }^{8}$

Beyond these two studies, data characterizing refractive and AL profiles in patients with FECD is scarce, and to our knowledge, no study has documented ALs in FECD patients using optical biometry. As such, the purpose of this study is to investigate the association between FECD and axial hypermetropia and examine whether a correlation exists between shorter ALs and corneal decompensation. In doing so, we hope to contribute more extensive and precise data to better characterize these clinical profiles.

\section{Materials and methods}

In this retrospective cohort study, selection involved 51 consecutive patients with FECD who underwent Descemet's stripping endothelial keratoplasty or penetrating keratoplasty, combined with cataract removal by a single corneal surgeon (JM), based at a cornea clinic in Sydney, Australia between July 2010 and March 2014. Controls were 58 patients randomly selected from those undergoing cataract surgery alone, by the same surgeon (JM), throughout the same duration of time as the FECD cohort. A process of simple random selection was used by compiling and numbering a list of all cataract surgeries between July 2010 and March 2014, and using a random number generator software. Patients with FECD or other corneal pathology were excluded from the control cohort. The study adhered to the tenets of the Declaration of Helsinki.

Eleven eyes of nine patients in the FECD group and 10 eyes of 5 patients in the control group were excluded from analysis due to history of previous corneal 
transplant, previous cataract surgery or inadequate data. The remaining 91 eyes of 50 patients with FECD and 110 eyes of 55 controls were included for analysis.

Medical records were reviewed, including preoperative clinical notes, optical biometry results, pentacam results and operation report. No postoperative data was utilized. Averages of measurements from both eyes were used for analysis.

The main outcome measures included $A L, A C D$ and spherical equivalent (SE). $A L$ and $A C D$ measurements were obtained from optical biometry (IOLMaster, Carl Zeiss Meditec, Dublin, CA, USA). If possible, SE was calculated using whichever parameters for refraction were available in the clinical notes, which included a mixture of subjective and auto-refraction. Other outcome measures included central corneal thickness (thinnest), uncorrected visual acuity, intraocular pressure (IOP) and keratometry.

Statistical analysis was performed using the SPSS software, version 22.0 (IBM/ SPSS Inc., Chicago, IL, USA). Independent sample t-tests were used to compare AL, ACD and SE. Chi-squared test was used to compare proportions of hypermetropic $\mathrm{SE}$ in each group. Simple linear regression analysis was used to correlate $\mathrm{AL}$ and corneal thickness.

\section{Results}

This study compared preoperative clinical measurements of 91 eyes of 50 FECD patients with 110 eyes of 55 controls. The baseline patient demographic is shown in Table 1. There was no difference in baseline age (69.6 vs 66.9 years, $p>0.05$ ) or keratometry ( 43.66 vs $43.09, p>0.05)$. The FECD group had a greater proportion of females (70\% vs $53 \%)$, more astigmatism (1.67D vs $1.02 \mathrm{D}, \mathrm{p}<0.01)$ and lower uncorrected visual acuities $(1.14$ vs $0.89, p<0.01)$ compared with controls.

Mean, standard deviation (SD) and confidence intervals (Cls) for AL, ACD and SE are presented in Table 2. Strong evidence of an association was found between FECD and AL. The mean AL of FECD patients ( $23.6 \mathrm{~mm} \mathrm{SD} \pm 0.86)$ was lower than controls (24.7 $\mathrm{mm} \mathrm{SD} \pm 1.76)$, with a mean difference of $1.1 \mathrm{~mm}(95 \% \mathrm{Cl}[0.57-1.63$ $\mathrm{mm}])(\mathrm{t}=4.12$ with 81 degrees of freedom [df], $p<0.001$, independent sample t-test).

FECD patients were also found to have shallower ACD. The mean ACD of FECD patients ( $3.0 \mathrm{~mm} \mathrm{SD} \pm 0.5$ ) was shorter than controls ( $3.3 \mathrm{~mm} \mathrm{SD} \pm 0.4)$, with a difference of $0.32 \mathrm{~mm}(95 \% \mathrm{Cl}$ [0.17-0.50]) ( $\mathrm{t}=3.79$ with $101 \mathrm{df}, \mathrm{p}<0.001$, independent sample t-test).

Refractive data was available for 22 FECD patients and 36 controls. Of these, 11 FECD patients (50\%) had hypermetropic refraction with a mean SE of $+1.40 \mathrm{D}$ (SD \pm 0.86$) ; 16$ controls (44\%) had hypermetropic refraction. Although the proportion was higher in the FECD group, this was not statistically significant $\left(x^{2}=0.17\right.$ with $1 \mathrm{df}, p=0.68$, chi-squared test). The overall mean SE of FECD patients (+0.10D 
Table 1. Comparison of baseline characteristics between FECD and control groups

\begin{tabular}{|l|l|l|l|}
\hline Variable & $\begin{array}{l}\text { FECD mean } \\
(\mathbf{n}=\mathbf{5 0})\end{array}$ & $\begin{array}{l}\text { Control mean } \\
(\mathbf{n}=\mathbf{5 5})\end{array}$ & P-value $^{\mathrm{a}}$ \\
\hline Age (years) & 69.6 & 66.9 & 0.21 \\
\hline $\begin{array}{l}\text { Sex } \\
\text { Male }\end{array}$ & $\begin{array}{l}35(70 \%) \\
15(30 \%)\end{array}$ & $\begin{array}{l}29(53 \%) \\
26(47 \%)\end{array}$ & \\
\hline LogMAR VA $(95 \% \mathrm{Cl})$ & $1.14(0.98-1.30)$ & $0.89(0.76-1.02)$ & $<0.01$ \\
\hline Keratometry $(95 \% \mathrm{Cl})$ & $43.66(43.19-44.13)$ & $43.09(42.53-43.65)$ & 0.27 \\
\hline Astigmatism $(95 \% \mathrm{Cl})$ & $1.67(1.31-2.02)$ & $1.02(0.85-1.18)$ & $<0.01$ \\
\hline IOP $(95 \% \mathrm{Cl})$ & $14.1(13.1-15.1)$ & $15.4(14.3-16.4)$ & $<0.01$ \\
\hline
\end{tabular}

andependent sample t-test

Table 2. Comparison of AL, ACD and SE between FECD and control groups

\begin{tabular}{|l|l|l|l|l|}
\hline Variable & FECD mean & Control mean & $\begin{array}{l}\text { Mean } \\
\text { difference } \\
{[95 \% \mathrm{Cl}]}\end{array}$ & P value $^{\mathrm{a}}$ \\
\hline $\mathrm{AL}(\mathrm{mm})$ & $\begin{array}{l}23.6 \pm 0.9 \\
(\mathrm{n}=49)\end{array}$ & $\begin{array}{l}24.7 \pm 1.8 \\
(\mathrm{n}=55)\end{array}$ & $1.1[0.5-1.6]$ & 0.001 \\
\hline $\mathrm{ACD}(\mathrm{mm})$ & $\begin{array}{l}3.0 \pm 0.5 \\
(\mathrm{n}=48)\end{array}$ & $\begin{array}{l}3.3 \pm 0.4 \\
(\mathrm{n}=55)\end{array}$ & $0.32[0.2-0.5]$ & 0.001 \\
\hline SE (D) & $\begin{array}{l}+0.1 \pm 1.6 \\
(\mathrm{n}=22)\end{array}$ & $\begin{array}{l}-1.3 \pm 3.5 \\
(\mathrm{n}=36)\end{array}$ & $1.4[0.1-2.8]$ & 0.036 \\
\hline
\end{tabular}

andependent sample t-test

$\mathrm{SD} \pm 1.57)$ was higher than controls $(-1.13 \mathrm{D} S \mathrm{SD} \pm 3.45)$, with a statistically significant difference of $1.43 \mathrm{D}(95 \% \mathrm{Cl}[-0.09$ to 2.76$])(t=2.15$ with $53 \mathrm{df}, \mathrm{p}=0.036$, independent sample t-test).

No statistically significant correlation was found between $A L$ and corneal thickness $\left(R^{2}=0.2 \%, \beta=-3.72 ; p=0.28\right.$, simple linear regression).

\section{Discussion}

With the use of non-contact imaging techniques, this study has provided strong evidence that patients with FECD have smaller than average eyes, with shorter $A L$ and shallower $A C D$ than controls. The main implication of these results is that 
shorter AL and ACD in FECD patients likely compound their risk of endothelial decompensation following phacoemulsification cataract surgery.

This is based on the premise that a shorter distance between ultrasound energy and the endothelium makes eyes more vulnerable to endothelial cell loss due to the closer proximity of heat and ultrasound energy, as well as movement of lens fragments and the risk of touch from surgical instruments. ${ }^{9}$ As such, shorter $A C D$ and $A L$ have been established as poor prognostic factors leading to greater endothelial cell loss as per Hwang et al. ${ }^{9}$ and Walkow et al. ${ }^{10}$ This underscores the importance of surgical planning and risk in FECD patients.

The refractive outcomes of this study were unfortunately less conclusive than our $A L$ and $A C D$ results, with a statistically higher mean SE in FECD group, yet no statistically significant difference in prevalence of refractive hypermetropia between groups. These results may be confounded by the presence of cataract, as increasing severity of cataract is known to induce myopic shift. ${ }^{11}$

The demographic profile of our cataract cohort seems to be consistent with data from previous large population-based cataract studies, in terms of age and the male-to-female ratio. ${ }^{12,13}$ Average $\mathrm{AL}$ in these cataract cohorts vary, with some reasonably shorter $(23.89 \pm 1.77$ [12]) than ours (24.7 mm SD \pm 1.76$)$. That said, other population-based studies also cite averages more consistent with our findings $\left(24 \pm 1.57^{13}\right)$.

These data may reflect an increasing prevalence of myopia in the community, for which there is an emerging body of evidence for, particularly in the Australasia region. ${ }^{14,15}$ Alternatively, our study results may also represent a higher-risk demographic of patients referred to the surgeon at hand, as our findings were based on a single surgeon cohort.

The main limitation of this study is the non-random sample of more severely affected FECD eyes, as selection was based on FECD patients who required corneal transplant. Given this bias, there is a possibility that the results reflect a potential tendency of FECD patients with shorter eyes to require surgery more often than other FECD patients. Pitts and Jay ${ }^{4}$ suggest this is not the case. They found no significant difference in $A L(p>0.05)$ or $A C D(0.05<p<1)$ between advanced FECD with corneal edema or previous surgery and those without edema, suggesting that both early and advanced FECD patients can be regarded as a uniform population. Nonetheless, a truly random sample of FECD patients remains ideal and would be recommended for further research.

The strengths of the study include the large sample size of the FECD cohort (particularly compared with previous studies on FECD patients) and use of average measurements from both eyes for each subject. This approach, as opposed to using data from each eye separately to boost sample size, has been shown to reduce the risk of type 1 error. $^{16}$ 
Our findings make a valuable contribution to the existing evidence, particularly in the profiling of FECD patients, with biometric data that has not yet been published in the literature. Given the continual advances in surgical outcomes of lamellar transplant, a close focus on prognostic factors and surgical considerations remains imperative.

\section{Acknowledgements}

None of the authors received any funding or financial support.

\section{References}

1. Park CY, Lee JK, Gore PK, Lim CY, Chuck RS. Keratoplasty in the United States: a 10-year review from 2005 through 2014. Ophthalmology. 2015;122(12):2432-2442. doi: 10.1016/j. ophtha.2015.08.017.

2. De Sanctis $U$, Alovisi $C$, Bauchiero $L$, et al. Changing trends in corneal graft surgery: a ten-year review. Int J Ophthalmol. 2016;9(1):48-52. doi: 10.18240/ijo.2016.01.08.

3. Elhalis H, Azizi B, Jurkunas UV. Fuchs endothelial corneal dystrophy. Ocul Surf. 2010;8(4):173-184.

4. Pitts JF, Jay JL. The association of Fuch's corneal endothelial dystrophy with axial hypermetropia, shallow anterior chamber, and angle closure glaucoma. Br J Ophthalmol. 1990;74:601-604.

5. Lowenstein A, Geyer O, Hourvitz D, Lazar M. The association of Fuchs corneal endothelial dystrophy with angle closure glaucoma. [Letter to Ed] Br J Ophthalmol. 1991;75(8):510.

6. Shen $P$, Zheng $Y$, Ding $X$, et al. Biometric measurements in highly myopic eyes. J Cataract Refract Surg. 2013;39(2):180-187. doi: 10.1016/j.jcrs.2012.08.064.

7. Sahin A, Hamrah P. Clinically relevant biometry. Curr Opin Ophthalmol. 2012;23(1):47-53. doi: 10.1097/ICU.0b013e32834cd63e.

8. Nakhli FR. Comparison of optical biometry and applanation ultrasound measurements of the axial length of the eye. Saudi J Ophthalmol. 2014;28(4):287-291. doi: 10.1016/j.sjopt.2014.04.003.

9. Hwang HB, Lyu B, Yim HB, Lee NY. Endothelial cell loss after phacoemulsification according to different anterior chamber depths. J Ophthalmol. 2015;2015:21076. doi: 10.1155/2015/210716.

10. Walkow T, Anders N, Klebe S. Endothelial cell loss after phacoemulsification: relation to preoperative and intraoperative parameters. J Cataract Refract Surg. 2000;26(5):727-732.

11. Samarawickrama C, Wang JJ, Burlutsky G, Tan AG, Mitchell P. Nuclear cataract and myopic shift in refraction. Am J Ophthalmol. 2007;144(3):457-459.

12. De Bernardo M, Zeppa L, Forte R, et al. Can we use the fellow eye biometric data to predict IOL power? Semin in Ophthalmol. 2017;32(3):363-370. doi: 10.3109/08820538.2015.1096400.

13. Covert DJ, Henry CR, Koenig SB. Intraocular lens power selection in the second eye of patients undergoing bilateral, sequential cataract extraction. Ophthalmology. 2010;117:49-54. doi: 10.1016/j.ophtha.2009.06.020.

14. Rose K, Smith W, Morgan I, Mitchell P. The increasing prevalence of myopia: implications for Australia. Clin Exp Ophthalmol. 2001;29(3):116-120.

15. Pan CW, Boey PY, Cheng CY, et al. Myopia, axial length, and age-related cataract: the Singapore Malay eye study. Invest Ophthalmol Vis Sci. 2013;54(7):4498-4502. doi: 10.1167/iovs.13-12271.

16. Armstrong RA. Statistical guidelines for the analysis of data obtained from one or both eyes. Ophthalmic Physiol Opt. 2013;33(1):7-14. doi: 10.1111/opo.12009. 